\title{
EXPERIENCIAS \\ INVESTIGATIVAS DESDE LA \\ AMAZONIA COLOMBIANA \\ PARA LA CONSTRUCCIÓN \\ DE POLITITICAS CONTRA \\ EL CAMBIO CLIMÁTICO: \\ PAGOS POR SERVICIOS \\ AMBIENTALES
}

\section{RESUMEN}

Investigaciones sobre los Pagos por Servicios Ambientales (PSA) buscan incorporar el valor potencial de los servicios ecosistémicos, tales como los sumideros de carbono, la protección de fuentes hídricas y biodiversidad, que se encuentran inmersos en tierras rurales. En el marco de los objetivos de desarrollo sostenible, la comunidad científica en la Amazonia tiene el reto de emprender investigaciones con sentido social para sensibilizar a los productores de sistemas productivos rurales sobre adopción de tecnologías orientadas a incorporar los principios de sostenibilidad en el campo amazónico colombiano, dada la importancia económica y cultural para la región. Se presentan avances de investigaciones sobre la estimación del costo de oportunidad del uso del suelo con amplio potencial para el diseño de política ambiental, estrategia para combatir el cambio climático, caso concreto: identificación de un valor para IOS PSA, de conformidad con el Decreto 0953 de 2013 y la Ley 99 de 1993. 


\section{YELLY YAMPARLI PARDO ROZO* \\ JAIME ENRIQUE VELÁSQUEZ RESTREPO** \\ MILTON CÉSAR ANDRADE ADAIME ${ }^{* * *}$}

Palabras clave: Pago por Servicios Ambientales, Cambio climático, Política ambiental, Amazonia colombiana, Fijación de carbono.

\section{RESEARCH EXPERIENCES FROM THE COLOMBIAN AMAZONIA TO BUILD RULES AGAINST CLIMATE CHANGE: PAYMENT FOR ENVIROMENTAL SERVICES}

\section{ABSTRACT}

Payment for Environmental Services (PSA) research seeks to incorporate the potential value of ecosystems services such as carbon sinks, protection of water sources and biodiversity, immersed in rural land. In the context of sustainable development goals, the university community in the Amazonia has challenged to undertake research with social sense to sensitize producers of rural production systems in the region, on adoption aimed at incorporating the principles of sustainability technologies in the Colombian Amazon area, given the economic and cultural importance for the region. Advances in research on estimating the opportunity cost of land use in the Amazon with great potential for the design of environmental policy, strategy to tackle climate change, concrete case: identification of a value for PSA in accordance with decree 0953 of 2013 and Law 99 of 1993, are presented.

Keywords: Payments for Environmental Services, Climate Change, Environmental Policy, Colombian Amazon, Carbon Fixation.

\section{INTRODUCCIÓN}

En coherencia con los principios filosóficos establecidos en los Objetivos de Desarrollo Sostenible (Naciones Unidas, 2015) y la norma ambiental colombiana, la formulación de políticas ambientales debe surgir del proceso de investigación científica (MADS, 2014). Dadas las potencialidades del contexto amazónico, considerado por su riqueza hídrica, paisajística y biodiversidad como uno de los ecosistemas estratégicos en el mundo, ante la problemática global de cambio climático, reta a las instituciones a generar producción académica pertinente y coherente con las necesidades en materia socioeconómica, política, cultural y ambiental.

* $\quad$ PhD (c), Profesora Investigadora. Universidad de la Amazonia. Colombia. Correo-e:y.pardo@udla.edu.co

* * PhD, Profesor Universidad de la Amazonia, Florencia, Caquetá. Colombia. Correo-e: jaimevere@hotmail.com

*** Magíster, Profesor Investigador Universidad de la Amazonia. Colombia. Correo-e:m.andrade@udla.edu.co

Recibido: 9 de septiembre de 2016, aceptado: 2 de diciembre de 2016.

Para citar el artículo: Pardo, Y. Y.; Velásquez, J. E.; Andrade, M. C.; (2016).

"Experiencias investigativas desde la Amazonia colombiana para la construcción de políticas contra el cambio climático: Pagos por Servicios Ambientales", en: Sotavento $M B A$, n. ${ }^{\circ} 28$, pp. 84-93.

DOI: http://dx.doi.org/10.18601/01233734.n28.09 
Una de las principales problemáticas regionales a abordar es la que se presenta en los suelos de la cuenca amazónica en Suramérica y que a escala se repite en la Amazonia colombiana: la deforestación y degradación de sus recursos naturales. Pese a su amplia vocación forestal, estos suelos han sido sometidos culturalmente a la introducción de pasturas y cultivos fuera de su aptitud de uso (Muñoz, 2007; Morton et al., 2006).

En el piedemonte amazónico caqueteño, la actividad agropecuaria desempeña un papel fundamental en la economía del departamento; no obstante, las prácticas tradicionales han suscitado daños ambientales a gran escala, como la deforestación (según el Sistema de Información Ambiental en Colombia del Ministerio de Medio Ambiente y Desarrollo Sostenible-MADS-, en 2014 la tasa de deforestación fue de 140 mil hectáreas por año entre 2011 a 2014, el más alto en América Latina), pérdida de suelos (por erosión y compactación), contaminación de fuentes hídricas (la alteración en la calidad, la disponibilidad de aguas y la reducción de su oferta) y el desplazamiento de especies selva adentro, entre otros (MAG, 2013; Tejo, 2003).

Esto ocurre debido a que las actividades agropecuarias representan el sustento de un porcentaje importante de hogares, dados los fuertes arraigos culturales introducidos desde 1900 en el panorama latinoamericano (FAO, 2013; Morton et al., 2006) y en la Amazonia colombiana (donde se genera cerca del 0,6\% del PIB colombiano pero que a nivel del Caquetá representa entre el 12 y $14 \%$ del PIB departamental). Luego de la industria del petróleo y sus derivados, que emiten cerca del $80 \%$ de los gases de efecto invernadero GEIs en el mundo y que han ocasionado el calentamiento global, la ganadería ostenta un segundo lugar en la emisión de estos gases (entre el $14 \%$ al $18 \%$ ), por lo cual surgen diversas estrategias y tecnologías para conseguir una armonización entre la productividad del campo, la rentabilidad y la disponibilidad de los recursos naturales y ambientales en el mediano y largo plazo (Andrade et al., 2014; Peters et al., 2013).

En este contexto, el documento tiene como objetivo presentar algunas de las investigaciones en la Amazonia para la construcción de políticas ambientales dirigidas al sector agropecuario, las cuales incorporan el valor económico potencial de los servicios ecosistémicos inmersos en las tierras productivas como parte de las estrategias para combatir el cambio climático desde la región; caso concreto, los estudios que se enfocan al análisis para establecer esquemas de pago por servicios ambientales PSA. Estos esfuerzos investigativos son consecuentes con los estudios prospectivos consignados en el Plan Estratégico de Ciencia, Tecnología e Innovación para el Caquetá ( Peña et al., 2012).

\section{FUNDAMENTO TEÓRICO}

Los Servicios Ecosistémicos ( $\mathrm{SE}$ ) se definen como mecanismos naturales que garantizan la disponibilidad de materias primas, energía y el ciclaje para la nueva disposición de estos recursos para satisfacer necesidades humanas; pueden clasificarse en servicios de apoyo, de aprovisionamiento, de regulación y servicios culturales (González, 2012, p. 112).

LOS SE no tienen establecido un mercado convencional, pero eso no significa que carezcan de precios; lo que sí es claro es la complejidad de establecerlos y sustentarlos (Labandeira, 2007; Freeman III, 2003). Ante la problemática de cambio climático, el SE de mayor interés es la captura de carbono, campo en el que se han generado oportunidades y respaldo mundial mediante los mecanismos de desarrollo limpio que permite la transacionalidad entre países para el cumplimiento de las metas de reducción planteadas desde el Protocolo de Kioto en 2000 , en los objetivos de desarrollo 
del milenio (2000-2015) y en las metas futuras en los objetivos de desarrollo sostenible a 2030. Los mercados que pueden establecerse son: la captura de carbono o reducción de emisiones evitadas; los servicios hidrológicos, como producción de agua, la conservación de fuentes hídricas, y los mercados ambientales de biodiversidad.

En predios rurales, se identifican SE importantes, como el secuestro de carbono, la conservación de bosques y la disponibilidad de agua, dadas las coberturas en bosques, pasturas, rastrojos y cultivos (Andrade et al., 2014; Somarriba et al., 2013). La importancia de la valoración de los SE en los predios agropecuarios radica en observar la contribución en el precio de las tierras, en la producción o en la utilidad y porque constituyen una línea base en la determinación de políticas ambientales (Pardo y Sanjinés, 2014, p. 144). Una aproximación es la estimación de los costos de oportunidad del uso de recursos.

En su papel normativo, la economía ambiental brinda teorías, métodos y técnicas para valorar los SE, para el diseño de los instrumentos económicos de regulación ambiental (tasas, impuestos y subsidios), y vía cantidades, los estándares o mando y control (Kolstad, 2001). Entre los mecanismos vía precios para apoyar el desarrollo de política pública, surge la fiscalidad ambiental, definida por González (2012, p. 114) como las contribuciones hacia el sistema jurídico tributario para coadyudar a la gestión ambiental, el cual se sustenta en la extrafiscalidad, el doble dividendo e impuestos verdes.

En Colombia, el Decreto 0953 de 2013 establece el PSA, definido como una transferencia de recursos financieros de las personas que se benefician por loS SE hacia los proveedores que los generan y que apoyan las externalidades positivas. Wunder et al. (2007, p. 125) los definen como una retribución por garantizar las condiciones de los procesos ecológicos que mantienen las actividades humanas, figura que presenta características tanto de mando y control como de incentivo económico.

Entre las principales experiencias de la valoración de SE en América Latina, autores como Larqué-Saavedra et al. (2004), de Robledo (2003), Escobar y Erazo (2006), citados en Forero, Pardo y Andrade (2016), estiman que una disponibilidad a pagar DAP por parte de la comunidad por hectárea en bosque (generador de un SE) se encuentra desde $\$ 47.800$ hasta $\$ 70.500$ al año, con un PSA de $\$ 842.000$ por hectárea para el productor; mientras que en el estudio de Aguilar y Marín (2015) en una zona del Caquetá, el DAP de la comunidad se encuentra entre $\$ 1.500$ a $\$ 6.500$ por conservar el recurso natural que garantiza el SE con un PSA para el productor de \$3'332.200 por hectárea, en promedio.

\section{METODOLOGÍA}

El estudio es de naturaleza descriptiva y exploratoria, con análisis de variables cualitativas y cuantitativas. Las unidades de análisis son las investigaciones con las siguientes características: i) constituyen información insumo o línea base directa para el diseño de política pública; ii) son interdisciplinarias, pues conjugan saberes de ciencias puras y sociales, por lo que sus unidades de estudio se sujetan al diseño experimental y observacional; iii) en materia de localización, los estudios se circunscriben al territorio amazónico; iv) fueron evaluados por pares externos inscritos en Colciencias; v) se apoyan en métodos cuantitativos aplicados; vi) las investigaciones pertenecen a las líneas de investigación en Desarrollo Sustentable (del programa de Doctorado en Ciencias Naturales y Desarrollo Sustentable DCNDS y la Maestría en Sistemas Sostenibles de Producción y Agroforestería); Fiscalidad Ambiental (de la Maestría en Tributación); Ambiente y Organización, del 
Programa Administración de Empresas de la Universidad de la Amazonia.

Para realizar el análisis de las contribuciones en materia de política ambiental, se utilizó la triangulación como método de análisis cualitativo, mediante el uso de varias fuentes de datos, teorías, investigaciones y ambientes de estudio que abordan un fenómeno (Benavides y Gómez, 2005 p. 15). Como técnica de recolección de datos de fuentes primarias, se realizaron entrevistas personalizadas con los investigadores principales; charlas con expertos en el tema; como fuentes secundarias se tomaron los proyectos y avances de las investigaciones presentadas en la Tabla 1, aplicando muestreo no probabilístico por conveniencia (Hernández et al., 2014; Jany, 1994).

Tabla 1. Investigaciones en la línea de desarrollo sustentable

Título, autor, año, estado

Valoración de la sostenibilidad en sistemas agropecuarios del piedemonte amazónico caqueteño (Pardo, 2016)*.

Valoración socio-económica del almacenamiento de carbono como insumo para la planificación del territorio de una zona piloto en Caquetá (Orjuela, 2016)*.

Valoración económica de la huella de carbono en sistemas de producción de caucho (Hevea brasilensis) como servicio ambiental en la Amazonía (Bahamón, 2015)**.

Fuente: Vicerrectoría de Investigaciones Uniamazonía.

*Estado en ejecución; **Terminado.

Tabla 2. Investigaciones en la línea de fiscalidad ambiental, ambiente y organizaciones

Título, autor, año, estado

Determinación de los Costos de Oportunidad del Uso del Suelo relativo al Servicio Ambiental Almacenamiento de Carbono en la microcuenca de la quebrada La Mono, en Belén de los Andaquíes, Caquetá (Pardo y Orjuela 2016)**.

Valoración Económica de las coberturas boscosas en sistemas productivos en San Vicente del Caguán, Caquetá (Forero et al.,2016)**.

Identificación del pago por servicios ambientales captura de carbono en sistemas ganaderos de la microcuenca La Mono en el municipio de Belén de los Andaquíes" (Artunduaga y Escobar, 2016)*

Fuente: Vicerrectoría de Investigaciones Universidad de la Amazonía.

*En ejecución; **Terminado.
El análisis se realizó en tres fases: 1) identificación de los productos académicos obtenidos y esperados de las investigaciones, 2) revisión literaria sobre IoS PSA y 3) Identificación de la contribución de la información para la construcción de política mediante la percepción de investigadores y expertos.

\section{RESULTADOS}

Los primeros aportes en la estimación de secuestro de carbono en sistemas agropecuarios en el piedemonte amazónico inicia desde 2000, con los estudios de Ibrahim et al. (2007) y Amézquita et al. (2008), quienes demostraron que los bosques nativos secuestran $30 \mathrm{tn} / \mathrm{ha}$ de carbono más que otro uso del suelo; y que de 182,9 Tn/ ha/ $1 \mathrm{~m}$-equivalente en topografía plana, la proporción de fijación de carbono fue 58,53\% en el suelo y $41,5 \%$ en la biomasa aérea.

Además, los mismos autores evidenciaron que el mejoramiento de pasturas y el aumento de coberturas arbóreas permiten fijar altos valores de carbono, donde el potencial de las fincas ganaderas se puede lograr mediante el incremento de plantaciones forestales y la liberación de tierras no aptas para la producción agropecuaria, para la regeneración natural de bosques secundarios. Este tipo de estudios estiman la huella ecológica de los sistemas agropecuarios, la cual es un indicador de la presión antrópica en los ecosistemas y la biodiversidad (Ibarra y Monroy, 2014; Doménech, 2010).

Para 2015, el estudio de Bahamón, titulado "Huella de carbono en sistemas agroforestales en el piedemonte amazónico", cuantificó las emisiones de C generadas en el manejo de los sistemas de producción de caucho en agroforestería con copoazú. Como principales resultados, obtuvo las emisiones generadas por la ganadería ( 1.263 toneladas de carbono/año/ finca) y las emisiones por fertilización $(0,15$ tn/año/finca). 
De otro lado, en la línea de investigación desarrollo sustentable, en 2016 se encuentra el estudio de Orjuela denominado "Valoración socio-económica del almacenamiento de carbono como insumo para la planificación del territorio de una zona piloto en Caquetá" con los siguientes objetivos: 1 ) estimar la capacidad de captura de carbono de los principales usos del suelo; 2) cuantificar los efectos de la transformación del paisaje sobre la captura de carbono y 3) evaluar la viabilidad socioeconómica del SE por parte de productores. La finalidad es la reorientación de sistemas productivos regionales para la planificación del territorio, que definan un balance positivo a favor de la captura de carbono como estrategia contra el cambio climático.

Como complemento, se cuenta con el estudio de Pardo (2016) sobre "Valoración de la sostenibilidad en sistemas agropecuarios del piedemonte amazónico", el cual tiene como objetivo proporcionar una nueva medición de la sostenibilidad en los predios agropecuarios a partir de la construcción de un indicador sostenibilidad, que integra variables en las dimensiones económicas, sociales, políticas y ambientales, partiendo de un análisis fundamentado en el modelamiento econométrico.

La principal contribución de este trabajo es la inclusión del valor económico de los SE, tales como el almacenamiento de carbono y la protección de fuentes hídricas; se fundamenta e integra disciplinas y ramas de la ciencia, como la economía ambiental y los sistemas sostenibles de producción.

El estudio tiene como productos: 1) el indicador de sostenibilidad en las fincas; 2) la estimación de la disponibilidad a aceptar por parte de los productores que pueden ser traducidas como costos de oportunidad de uso del suelo en diversas coberturas, y 3) la estimación de los beneficios económicos potenciales de la comunidad por contar con SE, a partir del cálculo de la variación equivalente como aproximación a la disponibilidad a recibir total (Pardo et al., 2012).

En la misma línea se encuentra el estudio "Determinación de los costos de oportunidad del uso del suelo relativo al servicio ambiental almacenamiento de carbono en la microcuenca La Mono en Belén de los Andaquíes, Caquetá" (Pardo y Orjuela, 2016), el cual tiene por objeto identificar el costo de oportunidad de uso del suelo con relación al SE almacenamiento de carbono, partiendo del cálculo de la productividad de la finca y aplicando el método de valoración de precios hedónicos, para observar si los SE inmersos en los sistemas de producción rural reflejan un valor en el mercado de tierras o constituyen una externalidad negativa.

En la línea de investigación de Fiscalidad Ambiental, se cuenta con el estudio denominado "Valoración económica de las coberturas boscosas en sistemas productivos en San Vicente del Caguán, Caquetá", presentado por Forero et al. (2016), en el que se emplearon métodos de valoración económica ambiental (valoración contingente y precios hedónicos), para corroborar los PSA presentados en el trabajo de Aguilar y Marín (2015) en la misma zona.

Los resultados indican que los bosques y pastos en las fincas tienen una disponibilidad a pagar marginal (DAPMg) positiva ( $\$ 842.000$ y $\$ 1^{\prime} 100.000$, respectivamente), de lo cual se infiere que estas coberturas aumentan el valor de la finca; en contraste, los cultivos y la productividad por hectárea no mostraron incidencia en el mercado de tierras. Este estudio, aunque corrobora la existencia de un mercado por SE que puede reflejarse en el precio de la tierra, difiere de los valores del PSA por hectárea presentados en Aguilar y Marín (2015) en la misma zona, quienes calcularon el valor económico del bosque ( $\$ 3^{\prime} 383.200$ ).

En otro estudio titulado "Identificación del pago por servicios ambientales captura de carbono en sistemas ganaderos en el municipio de Belén de los Andaquíes" presentado 
por Artunduaga y Escobar (2016), se busca determinar un valor como acercamiento a un pago por servicio ambiental almacenamiento de carbono como una nueva aplicación para el Decreto 0953 de 2013. Como avance de resultados a la fecha en la función hedónica lineal, se observa una relación positiva entre el precio de la finca y las coberturas boscosas con una DAPMg de $\$ 1^{\prime} 350.000$ por hectárea a una significancia del $22 \%$ y $\mathrm{R}^{2}=68 \%$; se espera mejorar la bondad de ajuste, mediante modelos de regresión no lineal Box Cox.

Los estudios citados plantean directamente la identificación de un valor para IOS PSA en la región, empleando métodos como los costos de oportunidad de uso del suelo; valoración del bosque; precios hedónicos y valoración contingente. Estos resultados proveen datos para realizar análisis costo-beneficio para sustentar la ejecución de proyectos o programas ambientales financiados desde el presupuesto de los planes de desarrollo municipal, o mediante la presentación de proyectos con organizaciones de cooperación internacional.

\section{CONCLUSIONES Y DISCUSIÓN}

Existen experiencias y desarrollos sobre la identificación de PSA en el contexto sur Andino Amazónico; con esta serie de estudios, la ciencia y el Estado buscan el reconocimiento del potencial económico que tienen recursos como la flora, la fauna, el agua, así como los servicios ambientales y mineros.

En los estudios citados se entregan los siguientes aportes para el diseño de política: 1) la cuantificación del almacenamiento de carbono en toneladas en las coberturas (bosques, pastos y cultivos); 2) la contribución marginal de loS SE almacenamiento de carbono y protección de fuentes hídricas al valor de la hectárea de las fincas agropecuarias; 3 ) la contribución económica de las coberturas al valor del predio a través de la DAPMg; 4) la identificación y comparación del costo de oportunidad del uso de la tierra en los estudios, y 5) la DAP de la comunidad por los beneficios de los SE y el esquema de pagos que deberá realizarse para formularlos.

Los autores de estos estudios coinciden en afirmar que las investigaciones deben trascender hacia la consolidación de política pública; esto es, fortalecer la triada EmpresaUniversidad-Estado, estrategia planteada desde los planes de desarrollo a partir de 2001 y que aún está lejos de consolidarse por la lentitud, rezago y distanciamiento entre la investigación y la formulación de políticas públicas; luego, indican que el esquema de los PSA debe surtir las siguientes fases: 1 ) contextualización (que implica identificación de la zona y de los actores), 2) identificación de las área objeto de estudio e influencia en el recurso, 3) la construcción de instrumentos para determinar el valor económico del bosque o los costos de oportunidad de uso del mismo, 4) la formulación del esquema del PSA con la información - línea base y 5) aprobación de la política pública y divulgación (lo que supone un acto administrativo con claridad en la financiación y cronograma de pagos).

Para ello, las investigaciones requieren integrar los resultados de los estudios propuestos; divulgar y socializar los resultados a la comunidad y a funcionarios de gobierno (alcaldes, gobernadores, concejales, diputados), y consolidar estas políticas mediante ordenanzas y organismos de control para lograr la trasferencia en programas tecnológicos, capacitaciones, participación en proyectos por parte de la comunidad campesina o propietarios de tierras.

Las experiencias investigativas -desarrolladas y en fase de ejecución- bajo las diversas líneas de investigación y de diferentes instituciones científicas proveen una línea base 
para la construcción de política pública; los esfuerzos en materia de inversión y financiación de recursos, formación de talento humano e infraestructura, responden en el mediano y largo plazo, a la instauración de una cultura frente a la sostenibilidad, como metas para el departamento.

Las investigaciones sustentan el diseño de políticas ambientales, ya sea mediante mecanismos de mando y control (como los estándares) o los instrumentos económicos (como impuestos, tasas, subsidios), o mecanismos mixtos, como es el caso de IoS PSA, figura hacia la cual se están enfocando los estudios de política pública, que favorecen la conservación del inventario forestal que puede cumplir tres tareas importantes: regulación hídrica, fijación de carbono y conservación de la biodiversidad.

Existe viabilidad normativa y jurídica en materia de instauración de mecanismos de regulación y políticas; se cuenta con los estudios interdisciplinarios que van desde lo técnico, lo tecnológico, lo financiero, lo económico y lo tributario, para incorporar los principios de desarrollo sostenible, y así preservar los recursos naturales y ambientales, mitigar el cambio climático, y buscar un desarrollo y calidad de vida a partir de las ventajas comparativas y competitivas de esta región.

La principal recomendación de este ejercicio es encadenar las iniciativas y los resultados de las investigaciones con otras instituciones científicas, corporaciones y organizaciones no gubernamentales, para fortalecer resultados y corroborar las perspectivas a fin de convertir la producción académica y científica en política pública materializada en planes, programas y proyectos, generadores de cultura ambiental para establecer un nuevo paradigma desde la Amazonia colombiana, que apunte a las apuestas y cadenas productivas, que redunde en aumentos de bienestar para las comunidades.

\section{REFERENCIAS}

Aguilar, V. y Marín, A. (2015). Primeros pasos de pago por servicios ambientales en el departamento del Caquetá. Seminario de Fiscalidad Ambiental SIFA: Avances y perspectivas en el mundo. Universidad de la Amazonia, Florencia Caquetá.

Amézquita, M. C.; Amézquita, E.; Casasola, F.; Ramírez, B. L.; Giraldo, H.; Gómez, M. E.; Llanderal, T.; Velásquez, J. e Ibrahim, M. A. (2008). Chapter 3. C Stock and sequestration. En: T'Mannetje, L.; Amézquita, M. C.; Buurman, P. e Ibrahim, M. A. (eds.). Carbon sequestration in tropical grassland ecosystems. Holanda: Wageningen Academic Publishers, pp. 49-67.

Andrade, H.; Marín, L.y Pachón, D. (2014). Fijación de carbono y porcentaje de sombra en sistemas de producción de Café (Coffea arábica L.) en el Líbano, Tolima, Colombia. Bioagro 26(2). Universidad del Tolima, Ibagué, Colombia.

Artunduaga, L.y Escobar, Y. (2016). Identificación del pago por servicios ambientales captura de carbono en sistemas ganaderos de la microcuenca La Mono en el municipio de Belén de los Andaquíes. Propuesta de Tesis de grado, Maestría en Tributación, Universidad de la Amazonia.

Bahamón, 0. (2015). Valoración económica de la huella de carbono en sistemas de produccion de caucho (hevea brasilensis) como servicio ambiental en la Amazonía caqueteña. Universidad de la Amazonia, Tesis de maestría en Sistemas Sostenibles de Producción, Facultad de Ciencias Agropecuarias. Florencia, Caquetá.

Benadives M. y Gómez, C. (2005). Métodos en investigación cualitativa: la triangulación. Revista Colombiana de Psiquiatría, vol. XxxIv, n. ${ }^{\circ}$, pp. 118-124.

Congreso de Colombia, Ley 99 de 1993 (22/diciembre/93). Art. 1 y Art. 3. Creación del Ministerio de Medio Ambiente y Sistema Nacional Ambiental, República de Colombia.

Doménech, Q.J. L. (2010). Huella ecológica y desarrollo sostenible. España: AENOR - Asociación 
Española de Normalización y Certificación. Disponible en: http://www.ebrary.com

Forero, A.; Pardo, Y. y Andrade, M. (2016). Valoración económica de las coberturas boscosas en sistemas productivos en San Vicente del Caguán, Seminario Internacional de Investigación, Innovación y Competitividad, Una estrategia de desarrollo agroindustrial en Territorio de Paz (Uniamazonia, octubre de 2016), Florencia, Caquetá.

Freeman III, A. M. (2003). The measurement of environmental and resources values. Theory and methods. Second edition. Resources for the future. Washington D.C.

González, M. A. (2012). Pagos por Servicios Ambientales en la lucha contra la desertificación: esquemas de pagos por servicios ambientales. Editorial Académica Española.

Hernández, R.; Fernández, C. y Baptista, L. (2014). Metodología de la Investigación. Quinta Edición, México.

Ibarra, J. M. y Monroy, A. (2014). Cuestionario para calcular huella ecológica de estudiantes universitarios mexicanos y su aplicación en el campus Zaragoza de la Universidad Nacional. Disponible en: http://www.sciencedirect.com/science/article/ $\mathrm{pii} / \mathrm{S} 1405888 \times 14720893$

Ibrahim, M.; Chacón, M.; Cuartas, C.; Naranjo, J.; Ponce, G.; Vega, P.; Casasola, F.y Rojas, J. (2007). Almacenamiento de carbono en los suelos y biomasa arbórea en sistemas de uso de la tierra en paisaje ganadero de Colombia, Costa Rica y Nicaragua. Revista Agroforestería en las Américas n. ${ }^{\circ} 45$.

Jany, J. (1994). Investigación integral de mercados, un enfoque operativo. Colombia: Mc Graw Hill.

Kolstad, Ch. (2001). Economía ambiental. México: Oxford University Press.

Labandeira, X.; León, C. y Vázquez, M. X. (2007). Economía Ambiental. Madrid: Pearson educación S. A.

Ministerio de Agricultura y Ganadería, MAG. (2013). Ganadería sostenible y cambio climático. Aportes para el proceso de construcción de NAMA
(2013). Costa Rica: IICA, PNUD, Low Emissión Capacity building Programme, GIZ.

Ministerio de Medio Ambiente y Desarrollo Sostenible, MADS - Programa de Naciones Unidas para el Desarrollo, PNUMA (2014). V Informe nacional de biodiversidad de Colombia ante el convenio de diversidad biológica. Bogotá, D.C.

Morton, D.; Shimabukuro, E.; Anderson, L.; Freitas, R.y Morisette J. (2006). Cropland expansión changes deforestation dynamics in the southhern Brazilian Amazon Proc. Natl. Acad. Sci usA.

Muñoz, J. (2007). Contribución a la sostenibilidad de los núcleos familiares asentados en fincas del piedemonte amazónico colombiano. Tesis presentada en opción al grado científico de Doctor en Ciencias Agrícolas, Universidad Agraria de La Habana "Fructuoso Rodríguez Pérez", La Habana.

Naciones Unidas (2015). Objetivos de Desarrollo Sostenible. Asamblea General, 12 de agosto de 2015. Disponible en: www.un.org/es/comun/ docs/?symbol=A/69/L.85. Naciones Unidas (2015).

Organización de las Naciones Unidas para la Agricultura y Alimentación, FAO (2013). Enfrentando el cambio climático a través de la ganadería: una evaluación global de las emisiones y oportunidades de mitigación.

Orjuela, J. (2016). Valoración socio-económica del almacenamiento de carbono como insumo para la planificación del territorio de una zona piloto en Caquetá. Universidad de la Amazonia, Vicerrectoría de Investigaciones y Posgrados, Programa de Doctorado en Ciencias Naturales y Desarrollo Sustentable, Florencia, Caquetá.

Pardo, Y. Y.; Andrade, M. C. y Hermosa, D. (2012). Evaluación Económica de políticas y proyectos: métodos alternativos y estudios de caso. Cali Colombia: FERIVA.

Pardo, Y. y Orjuela, J. (2016). Determinación de los Costos de Oportunidad del Uso del Suelo relativo al Servicio Ambiental Almacenamiento de Carbono en la microcuenca de la quebrada $\mathrm{La}$ Mono en Belén de los Andaquíes, Universidad de la Amazonia, Florencia, Caquetá. 
Pardo, Y.; Forero, A. y Andrade, M. (2016). Valoración económica de las coberturas boscosas en sistemas productivos en San Vicente del Caguán, Caquetá. Ponencia Seminario Internacional de Ciencia y tecnología y Semana Alimentaria, Universidad de la Amazonia, octubre de 2016.

Pardo, Y. y Sanjines, G. (2014). Valoración Económica de servicios ambientales en sistemas agroforestales en América Latina. Revista de la Facultad de Ciencias Contables, Económicas y Administrativas FACCEA, Universidad de la Amazonia, Volumen 4, Número 2. Florencia, Caquetá.

Pardo, Y. (2016). Valoración de la sostenibilidad en sistemas agropecuarios del piedemonte amazónico. Universidad de la Amazonia, Vicerrectoría de Investigaciones y Posgrados, Programa de Doctorado en Ciencias Naturales y Desarrollo Sustentable, Florencia, Caquetá.

Peña, P.; Ríos, .G; Marles, C. y Espinosa, L. (2012). Plan estratégico de Ciencia y Tecnología e innovación para el Caquetá. Ordenanza n. ${ }^{\circ} 035$ de 2012. Asamblea Departamental del Caquetá.

Peters, M.; Herrero, M.; Fisher, M.; KarlHeinz, E.; Idupulapati, R.; Guntur, V.; Castro, A.;
Arango, J.; Chara, J.; Murgueitio, E. y Searchinger, T. (2013). Challenges and opportunidties for improvising eco-eficiency of tropical forage-based systems to mittigate greenhouse gas emissións. Cali, Colombia: Centro Internacional de Agricultura, CIAT.

República de Colombia. Decreto 0953 de 2013. El cual reglamenta el Artículo 111 de la Ley 99 de 1993. República de Colombia. Mayo de 2013.

Somarriba, E.; Cerda, R.; Orozco, L.; Cifuentes, M.; Dávila, H.; Espina, T.; Mavisoy, H.; Ávila, G.; Alvarado, E.; Poveda, M.; Astorga, C.; Saya, E. y Deheuvels, 0. (2013). Carbon stocks and cocoa yields in agroforestry systems of Central América. Agriculture, Ecosistems and Enviromental, Elsevier.

Tejo, P. (coord.) (2003). Mercado de tierras agrícolas en América Latina y el Caribe: Mercado de tierras agrícolas para América Latina. Libro Comisión Económica para América Latina CEPALGTZ. Santiago de Chile.

Wunder, S.; Wertz, Sh. y Moreno, R. (2007). Pago por servicios ambientales: una nueva forma de conservar la biodiversidad. Gaceta Ecológica, julio-diciembre, 39-52. 